TRANSACTIONS OF THE

AMERICAN MATHEMATICAL SOCIETY

Volume 359, Number 3, March 2007, Pages 1339-1350

S 0002-9947(06)03963-8

Article electronically published on October 16, 2006

\title{
SMALL BALL PROBABILITIES FOR THE SLEPIAN GAUSSIAN FIELDS
}

\author{
FUCHANG GAO AND WENBO V. LI
}

\begin{abstract}
The $d$-dimensional Slepian Gaussian random field $\left\{S(\mathbf{t}), \mathbf{t} \in \mathbb{R}_{+}^{d}\right\}$ is a mean zero Gaussian process with covariance function $\mathbb{E} S(\mathbf{s}) S(\mathbf{t}) \stackrel{+}{=}$ $\prod_{i=1}^{d} \max \left(0, a_{i}-\left|s_{i}-t_{i}\right|\right)$ for $a_{i}>0$ and $\mathbf{t}=\left(t_{1}, \cdots, t_{d}\right) \in \mathbb{R}_{+}^{d}$. Small ball probabilities for $S(\mathbf{t})$ are obtained under the $L_{2}$-norm on $[0,1]^{d}$, and under the sup-norm on $[0,1]^{2}$ which implies Talagrand's result for the Brownian sheet. The method of proof for the sup-norm case is purely probabilistic and analytic, and thus avoids ingenious combinatoric arguments of using decreasing mathematical induction. In particular, Riesz product techniques are new ingredients in our arguments.
\end{abstract}

\section{INTRODUCTION}

For a given continuous Gaussian random field $X(\mathbf{t}), \mathbf{t} \in[0,1]^{d}$, the small ball probability studies the asymptotic behavior of $\log \mathbb{P}(\|X\| \leq \varepsilon)$ as $\varepsilon \rightarrow 0^{+}$, where $\|\cdot\|$ is a norm on the space $C\left([0,1]^{d}\right)$. In the literature, small deviation probabilities of various types are studied and applied to many problems of interest under different names such as small ball probability, lower tail behaviors, two-sided boundary crossing probability, the first exit time, etc. The survey paper of Li and Shao LS01] for Gaussian processes, together with its extended references, covers much of the recent progress in this area. In particular, various applications and connections with other areas of probability and analysis are discussed.

Arguably, the most fundamental multi-parameter Gaussian random field is the so-called Brownian sheet $\left\{W(\mathbf{t}), \mathbf{t} \in \mathbb{R}_{+}^{d}\right\}$ with mean zero and covariance

$$
\mathbb{E} W(\mathbf{s}) W(\mathbf{t})=\prod_{i=1}^{d} \min \left(s_{i}, t_{i}\right),
$$

where $\mathbf{s}=\left(s_{1}, \cdots, s_{d}\right) \in \mathbb{R}_{+}^{d}$ and $\mathbf{t}=\left(t_{1}, \cdots, t_{d}\right) \in \mathbb{R}_{+}^{d}$. It is a tensor product of the standard Brownian motions. The first systematic study of the small ball probability for $W(\mathbf{t})$ and its tied-down variants with applications to KolmogorovSmirnov statistics and Chung's LIL is presented in Bass [Ba88, together with the sharp lower bound in (1.1) below, given also in Lifshits and Tsyrelson [LT86]. In a striking paper in comparison with the $L_{2}$-norm result in (1.3) for $d=2$, Talagrand

Received by the editors October 28, 2004 and, in revised form, February 2, 2005.

2000 Mathematics Subject Classification. Primary 60G15; Secondary 42A55.

The first author was supported in part by NSF Grants EPS-0132626 and DMS-0405855.

The second author was supported in part by NSF Grant DMS-0204513.

(C)2006 American Mathematical Society Reverts to public domain 28 years from publication 
Ta94 showed the sharp upper bound of the estimate that

$$
-C_{2} \varepsilon^{-2}|\log \varepsilon|^{3} \leq \log \mathbb{P}\left(\sup _{0 \leq s, t \leq 1}|W(s, t)| \leq \varepsilon\right) \leq-C_{1} \varepsilon^{-2}|\log \varepsilon|^{3}
$$

for $\varepsilon>0$ small. Here and throughout this paper, we use $C, C_{1}$ or $C_{2}$ to denote positive absolute constants which may change values from line to line. A somewhat simplified proof of the upper bound in (1.1) can be found in Dunker [Du00]. Here we will give a simple and general approach to avoid ingenious combinatoric arguments used before. Our method is purely probabilistic and analytic by providing explicit constructions via Riesz products. We shift the combinatorics difficulties in $d=2$ into the structure of analytic considerations. This is why the arguments in our proof are clear in ideas and can also be applied to Slepian Gaussian random field in $d=2$. Indeed, this is the main contribution of the paper, and it will be discussed in details in Section 2. In particular, Riesz product techniques are introduced as new ingredients in our arguments.

For $d \geq 3$, the situation becomes much more difficult, as the combinatorial arguments used in Talagrand [Ta94] for $d=2$ fail and our analytic method (which avoids combinatorics in $d=2$ ) also encounters interesting combinatorial difficulties which are somewhat simpler than before. We hope that the idea of shifting combinatorial difficulties into structures of analytic considerations can be carried out in the case $d \geq 3$. The best known bounds for $d \geq 3$ are

$$
-C_{2} \varepsilon^{-2}|\log \varepsilon|^{2 d-1} \leq \log \mathbb{P}\left(\sup _{\mathbf{t} \in[0,1]^{d}}|W(\mathbf{t})| \leq \varepsilon\right) \leq-C_{1} \varepsilon^{-2}|\log \varepsilon|^{2 d-2} .
$$

The upper bound above follows from (1.3), and the lower bound was later proved by Dunker, Kuhn, Lifshits, and Linde D-99 (a slightly weaker lower bound is given in Belinsky [Be98). It should be pointed out that the proofs of the lower bound in D-99] and Be98 are based on approximation theory and the connection between small ball probability and entropy numbers discovered in Kuelbs and Li [KL93, and hence are not probabilistic but analytic. Also, arguments to obtain small ball probabilities in Belinsky and Linde [BL02] for fractional Brownian sheets are based on fractional integration operators and hence are not probabilistic. It would be interesting to find a pure probabilistic proof of the lower bound in (1.2). The only known probabilistic proof for the lower bound with $d \geq 2$ is presented in Bass Ba88, which gives $3 d-3$ for the power of the log-term.

Before we finish the discussion on Brownian sheets, we need to mention the results under the much simpler $L_{2}$-norm given in Csáki [Cs82]. Namely,

$$
\log \mathbb{P}\left(\int_{\mathbf{t} \in[0,1]^{d}}|W(\mathbf{t})|^{2} d \mathbf{t} \leq \varepsilon^{2}\right) \sim-c_{d} \varepsilon^{-2}|\log \varepsilon|^{2 d-2},
$$

where $c_{d}=2^{d-2} /\left(\sqrt{2} \pi^{d-1}(d-1) !\right)$. Various generalizations of the above result are given in Li Li92, Karol, Nazarov and Nikitin [K-03], Fill and Torcaso [FT04, and Gao and Li GL04.

The main purpose of this paper is to introduce Riesz product techniques into the study of small ball probability under sup norm. It can also give a more transparent and much simpler proof when applied to 2-dimensional Brownian sheets, and in fact, we believe it is the simplest and most elementary proof for the upper bound. Our method is much more general and can also be applied to many related problems. The key is to avoid hard combinatorial arguments. The method is introduced 
through the small ball estimates for the so-called $d$-dimensional Slepian Gaussian random fields $\left\{S(\mathbf{t}), \mathbf{t} \in \mathbb{R}_{+}^{d}\right\}$ which is a mean zero Gaussian process with covariance function

$$
\mathbb{E} S(\mathbf{t}) S(\mathbf{s})=\prod_{i=1}^{d} \max \left(0, a_{i}-\left|s_{i}-t_{i}\right|\right)
$$

for $a_{i}>0$. Such fields are homogeneous and are tensor products of the few stationary one-parameter Gaussian processes for which exact distributions of certain functionals, such as maximum, are known. To be more precise, it is the tensor products of one-dimensional Slepian processes defined by

$$
S(t)=W(t+a)-W(t), \quad 0 \leq t \leq 1,
$$

with $a>0$ fixed, where $W(t)$ is the standard Brownian motion. See Adler Ad84 for a detailed study of the upper tail behavior for $S(\mathbf{t}), \mathbf{t} \in[0,1]^{d}$. Our first result is the following.

Theorem 1.1. We have for $\varepsilon>0$ small

$$
-C_{2} \varepsilon^{-2}|\log \varepsilon|^{3} \leq \log \mathbb{P}\left(\sup _{0 \leq s, t \leq 1}|S(s, t)| \leq \varepsilon\right) \leq-C_{1} \varepsilon^{-2}|\log \varepsilon|^{3} .
$$

The proofs and relations with Brownian sheets will be discussed in detail in Section 2.

Our next result concerns the $L_{2}$-norm which should be compared with (1.3). The proof is given in Section 3.

Theorem 1.2. Let $S(\mathbf{t})$ be a Slepian field with $a_{i} \geq 1$. Then

$$
\log \mathbb{P}\left(\int_{[0,1]^{d}}|S(\mathbf{t})|^{2} d \mathbf{t}<\varepsilon^{2}\right) \sim-k_{d} \varepsilon^{-2}|\log \varepsilon|^{2 d-2},
$$

where $k_{d}=2^{-d} c_{d}$ and $c_{d}$ is given in (1.3).

Finally, we have to point out that the estimate of small ball rates of Gaussian fields is important not only in probability theory, but also in other areas of mathematics. For example, through the relation between small ball probability and metric entropy, the small ball rates of Brownian sheets was applied to solve a long-standing open problem in approximation; see Kuelbs and Li [KL93].

The remainder of the paper is organized as follows. We prove Theorem $1.1 \mathrm{in}$ Section 2. The key analytical result is Theorem 2.1, which is proved by using Riesz products. This new approach has great potential of success in many other related problems; in particular for the $d \geq 3$ case in (1.2) which is under investigation. In Section 3, we prove Theorem 1.2 based on explicit Karhunen-Loéve expansion. Proposition 3.1 also provides the exact Laplace transform which is of independent interest.

\section{Proof of Theorem 1.1}

This is the main part of the paper on small ball estimates under sup norm for $d=2$. We first look at the lower bound in Theorem 1.1, which follows from the lower bound for Brownian sheets. Indeed, we can write in distribution

$$
S(s, t)=W\left(s+a_{1}, t+a_{2}\right)-W\left(s+a_{1}, t\right)-W\left(s, t+a_{2}\right)+W(s, t) .
$$


Note that

$$
\begin{aligned}
& \sup _{0 \leq s, t \leq 1}\left|W\left(s+a_{1}, t+a_{2}\right)-W\left(s+a_{1}, t\right)-W\left(s, t+a_{2}\right)+W(s, t)\right| \\
\leq & 4 \sup _{0 \leq s, t \leq 1+a}|W(s, t)|,
\end{aligned}
$$

where $a=\max \left(a_{1}, a_{2}\right)$, and thus

$$
\begin{aligned}
\log \mathbb{P}\left(\sup _{0 \leq s, t \leq 1}|S(s, t)| \leq \varepsilon\right) & \geq \log \mathbb{P}\left(4 \sup _{0 \leq s, t \leq 1+a}|W(s, t)| \leq \varepsilon\right) \\
& \geq-C_{2} \varepsilon^{-2}|\log \varepsilon|^{3},
\end{aligned}
$$

where the last line follows from (1.1). Furthermore, the above shows that the upper bound for $S(s, t)$ implies the upper bound for the Brownian sheet. Hence the Slepian field results obtained in Theorem 1.1 apply to the Brownian sheet for the much harder upper bound case.

We break the upper bound case into several steps in order to highlight the basic ideas. First, note that for any $0<\delta<1$,

$$
\mathbb{P}\left(\sup _{0 \leq s, t \leq 1}|S(s, t)| \leq \varepsilon\right) \leq \mathbb{P}\left(\sup _{0 \leq s, t \leq \delta}|S(s, t)| \leq \varepsilon\right)=\mathbb{P}\left(\sup _{0 \leq s, t \leq 1}|\widetilde{S}(s, t)| \leq \varepsilon \delta^{-1}\right)
$$

by the scaling properties of Slepian process, where

$$
\widetilde{S}(s, t)=W\left(s+\delta^{-1} a_{1}, t+\delta^{-1} a_{2}\right)-W\left(s+\delta^{-1} a_{1}, t\right)-W\left(s, t+\delta^{-1} a_{2}\right)+W(s, t) .
$$

Thus, it is enough to consider the case where $\min \left(a_{1}, a_{2}\right) \geq 1$ by taking $\delta \leq$ $\min \left(a_{1}, a_{2}\right)$.

Second, we use Anderson's inequality to concentrate on a suitable expansion for $S(s, t)$. Let $\Phi_{n}$ be an orthonormal basis of $L^{2}\left([0,1+a]^{2}\right)$ with $a=\max \left(a_{1}, a_{2}\right)$. Then by the definition of $S(s, t)$, we can express in distribution

$$
S(s, t)=\sum_{n=1}^{\infty} \int_{s}^{s+a_{1}} \int_{t}^{t+a_{2}} \Phi_{n}(x, y) d y d x \cdot g_{n}
$$

where $g_{n}$ are independent standard normal random variables. By Anderson's inequality, we have

$$
\mathbb{P}\left(\sup _{0 \leq s, t \leq 1}|S(s, t)| \leq \varepsilon\right) \leq \mathbb{P}\left(\sup _{0 \leq s, t \leq 1}|X(s, t)| \leq \varepsilon\right),
$$

where $X(s, t)$ is any partial sum in the representation (2.1) for $S(s, t)$. Of course, an explicit choice of $X(s, t)$ is defined below. We choose an orthonormal basis that contains a special set of orthonormal functions $\phi_{m, i, j}, 0 \leq m \leq n, 1 \leq i \leq 2^{m}$, $1 \leq j \leq 2^{n-m}$, such that the partial sum

$$
X_{n}(s, t):=\sum_{m=0}^{n} \sum_{i=1}^{2^{m}} \sum_{j=1}^{2^{n-m}} \int_{s}^{s+a_{1}} \int_{t}^{t+a_{2}} \phi_{m, i, j}(x, y) d y d x \cdot g_{m, i, j}
$$

is relatively easy to handle, where $g_{m, i, j}$ are independent standard normal random variables. We choose $\phi_{m, i, j}$ in such a way that

$$
\int_{s}^{s+a_{1}} \int_{t}^{t+a_{2}} \phi_{m, i, j}(x, y) d y d x=\left[\psi_{m, i}\left(s+a_{1}\right)-\psi_{m, i}(s)\right] \cdot\left[\psi_{n-m, j}\left(t+a_{2}\right)-\psi_{n-m, j}(t)\right],
$$


for $0 \leq s, t \leq 1$, where

$$
\psi_{m, i}(t)= \begin{cases}0 & t \notin\left((i-1) 2^{-m}, i 2^{-m}\right) \\ 2^{-m / 2-2} & t=(i-3 / 4) 2^{-m} \\ -2^{-m / 2-2} & t=(i-1 / 4) 2^{-m} \\ \text { linear } & \text { otherwise. }\end{cases}
$$

Of course this can be achieved by taking

$$
\phi_{m, i, j}(x, y)=2^{m / 2} \operatorname{sgn}\left(\cos \left(2^{m+1} \pi x\right)\right) \cdot 2^{(n-m) / 2} \operatorname{sgn}\left(\cos \left(2^{n-m+1} \pi y\right)\right)
$$

on the rectangle $\left[(i-1) 2^{-m}, i 2^{-m}\right] \times\left[(j-1) 2^{-(n-m)}, j 2^{-(n-m)}\right]$, and $\phi_{m, i, j}(s, t)=0$ otherwise. In addition, we have by using the fact that $\min \left(a_{1}, a_{2}\right) \geq 1$,

$$
X_{n}(s, t)=\sum_{m=0}^{n} \sum_{i=1}^{2^{m}} \sum_{j=1}^{2^{n-m}} \psi_{m, i}(s) \psi_{n-m, j}(t) \cdot g_{m, i, j}, \quad(s, t) \in[0,1]^{2},
$$

which follows from (2.2).

We choose such functions because of their orthogonality with the corresponding $h_{m, i}$ functions that will be used in our Riesz products. More precisely, we have $\int_{0}^{1} \psi_{m, i}(t) d t=0$, and if $h_{m^{\prime}, i^{\prime}}(t)$ is the scaled Haar function on $[0,1]$ given by $h_{-1,0}(t)=1$,

$$
h_{m^{\prime}, i^{\prime}}(t)=\left\{\begin{array}{rl}
1 & t \in\left[\left(i^{\prime}-1\right) 2^{-m^{\prime}},\left(i^{\prime}-1 / 2\right) 2^{-m^{\prime}}\right), \\
-1 & t \in\left[\left(i^{\prime}-1 / 2\right) 2^{-m^{\prime}}, i^{\prime} 2^{-m^{\prime}}\right), \\
0 & \text { otherwise, }
\end{array}\right.
$$

for $m^{\prime} \geq 0$ and $1 \leq i^{\prime} \leq 2^{m^{\prime}}$, then

$$
\int_{0}^{1} \psi_{m, i}(s) h_{m^{\prime}, i^{\prime}}(s) d s= \begin{cases}0 & \text { if } m^{\prime}<m \text { or } m^{\prime}=m+1, \\ 2^{-3 m / 2-3} & \text { if }(m, i)=\left(m^{\prime}, i^{\prime}\right), \\ 0 & \text { if } m^{\prime}=m \text { and } i^{\prime} \neq i, \\ c_{m, m^{\prime}, i, i^{\prime}} & \text { if } m^{\prime}>m+1,\end{cases}
$$

where $c_{m, m^{\prime}, i, i^{\prime}}$ is a constant such that $\left|c_{m, m^{\prime}, i, i^{\prime}}\right| \leq 2^{-2\left(m^{\prime}-m\right)-1} \cdot 2^{-3 m / 2}$.

The third step is crucial and is the meat of this paper. We have to compare it with the key combinatorial argument in Talagrand Ta94, which is the following result: If $q=9$, then for each $n \geq 1$, and each family of numbers $\left(\alpha_{m, i, j}\right)_{(m, i, j) \in T_{n}}$, where

$$
T_{n}=\left\{(m, i, j): 0 \leq m \leq n-1,0 \leq i<2^{q m}, 0 \leq j<2^{q(n-m)}\right\},
$$

we have

$$
\sup _{0 \leq s, t \leq 1} \sum_{(m, i, j) \in T_{n}} \alpha_{m, i, j} \psi_{m, i}(s) \psi_{n-m, j}(t) \geq 2^{-3 q n / 2-7} \sum_{(m, i, j) \in T_{n}}\left|\alpha_{m, i, j}\right| .
$$

This has also been used in several related problems; see Temlyakov Te95] and Martin Ma04. The role of the parameter $q$ in Talagrand's combinatorial inequality (2.5) is to ensure that the contribution of certain error terms is sufficiently small. The proof uses ingenious combinatoric arguments via decreasing mathematical induction which is hard to apply in general settings.

We will show the following by using Riesz products which are simple and clear. More importantly, our new approach via Riesz products can be used in other related problems. 
Theorem 2.1. For each $n \geq 1$, and each family of numbers $\left(\alpha_{m, i, j}\right)$,

$$
\sup _{0 \leq s, t \leq 1} \sum_{m=0}^{n} \sum_{i=1}^{2^{m}} \sum_{j=1}^{2^{n-m}} \alpha_{m, i, j} \psi_{m, i}(s) \psi_{n-m, j}(t) \geq 2^{-3 n / 2-16} \sum_{m=0}^{n} \sum_{i=1}^{2^{m}} \sum_{j=1}^{2^{n-m}}\left|\alpha_{m, i, j}\right| .
$$

Proof. Define the Riesz product

$$
R(s, t)=\prod_{m=0}^{n}\left(1+\sigma \sum_{i=1}^{2^{m}} \sum_{j=1}^{2^{n-m}} \varepsilon_{m, i, j} h_{m, i}(s) h_{n-m, j}(t)\right)
$$

where $\varepsilon_{m, i, j} \in\{-1,1\}$, and $0<\sigma<1$ is a constant to be determined later. Note that $R(s, t)$ depends on $n$. Later in the proof, we will use $R(s, t)$ for a suitably chosen $n$.

Because $\left|h_{m, i}(s) h_{n-m, j}(t)\right| \leq 1$, we have $R \geq 0$. Thus,

$$
\begin{aligned}
\|R\|_{1} & =\int_{0}^{1} \int_{0}^{1} R(s, t) d s d t \\
& =\int_{0}^{1} \int_{0}^{1} \sum \prod_{m=0}^{n}\left[\sigma \sum_{i=1}^{2^{m}} \sum_{j=1}^{2^{n-m}} \varepsilon_{m, i, j} h_{m, i}(s) h_{n-m, j}(t)\right]^{\delta_{m}} d s d t
\end{aligned}
$$

where the outmost summation in the integrand is over all choices of $\delta_{m} \in\{0,1\}$, $0 \leq m \leq n$. Since the integral (with respect to $s$ ) of a product of any distinct $h_{m, i}(s)$ functions vanishes, the only remaining term in the integral is the case $\delta_{m}=0$ for all $m$, and its value is one. So, we have $\|R\|_{1}=1$. Note that $\|R\|_{1}=1$ for any choices of $\varepsilon_{m, i, j}$. In particular, we will choose $\varepsilon_{m, i, j}=\operatorname{sgn}\left(\alpha_{m, i, j}\right)$.

For notational simplicity, we denote

$$
\begin{gathered}
\widetilde{H}_{m}=\sum_{i=1}^{2^{m}} \sum_{j=1}^{2^{n-m}} \varepsilon_{m, i, j} h_{m, i}(s) h_{n-m, j}(t), \\
Y_{n}(s, t)=\sum_{m=0}^{n} \sum_{i=1}^{2^{m}} \sum_{j=1}^{2^{n-m}} \psi_{m, i}(s) \psi_{n-m, j}(t) \cdot \alpha_{m, i, j} .
\end{gathered}
$$

Write

$$
R(s, t)=1+\sigma \sum_{m=0}^{n} \widetilde{H}_{m}+\sum_{\Delta} \prod_{m=0}^{n}\left[\sigma \widetilde{H}_{m}\right]^{\delta_{m}}
$$

where

$$
\Delta=\left\{\left(\delta_{m}\right)_{m=0}^{n}: \delta_{m} \in\{0,1\}, \sum_{m=0}^{n} \delta_{m} \geq 2\right\}
$$


Because $\|R\|_{1}=1$, we have

$$
\begin{aligned}
\sup _{0 \leq s, t \leq 1} Y_{n}(s, t) \geq & \int_{0}^{1} \int_{0}^{1} Y_{n}(s, t) R(s, t) d s d t \\
= & \int_{0}^{1} \int_{0}^{1} Y_{n}(s, t) d s d t+\sigma \sum_{m=0}^{n} \int_{0}^{1} \int_{0}^{1} Y_{n}(s, t) \widetilde{H}_{m} d s d t \\
& +\sum_{\Delta} \int_{0}^{1} \int_{0}^{1} Y_{n}(s, t) \prod_{m=0}^{n}\left[\sigma \widetilde{H}_{m}\right]^{\delta_{m}} d s d t \\
=: & I_{1}+I_{2}+I_{3} .
\end{aligned}
$$

It is clear that $I_{1}=0$ since the integral of $\psi_{m, i}$ over $[0,1]$ vanishes. To estimate $I_{2}$, note that $\psi_{m, i}(s) \psi_{n-m, j}(t)$ is orthogonal to $h_{m^{\prime}, i^{\prime}}(s) h_{n-m^{\prime}, j^{\prime}}(t)$ if $(m, i, j) \neq$ $\left(m^{\prime}, i^{\prime}, j^{\prime}\right)$, and hence we have

$$
\begin{aligned}
I_{2} & =\sigma \sum_{m=0}^{n} \sum_{i=1}^{2^{m}} \sum_{j=1}^{2^{n-m}} \varepsilon_{m, i, j} \int_{0}^{1} \int_{0}^{1} \psi_{m, i}(s) \psi_{n-m, j}(t) h_{m, i}(s) h_{n-m, j}(t) d s d t \cdot \alpha_{m, i, j} \\
& =2^{-3 n / 2-6} \sigma \sum_{m=0}^{n} \sum_{i=1}^{2^{m}} \sum_{j=1}^{2^{n-m}}\left|\alpha_{m, i, j}\right|
\end{aligned}
$$

where in the last equality we also used $(2.7)$ for the case $(m, i, j)=\left(m^{\prime}, i^{\prime}, j^{\prime}\right)$.

Now, we start to estimate $I_{3}$. For each $\left(\delta_{k}\right) \in \Delta$, let $p=\min \left\{k: \delta_{k}=1\right\}$ and $q=\max \left\{k: \delta_{k}=1\right\}$. So, $p<q$. For each fixed $(m, i, j)$, consider

$$
J_{m}:=\sum_{\Delta} \int_{0}^{1} \int_{0}^{1} \psi_{m, i}(s) \psi_{n-m, j}(t) \prod_{k=0}^{n}\left[\sigma \widetilde{H}_{k}\right]^{\delta_{k}} d s d t .
$$

If $q<m$, then, for each $t$ fixed,

$$
\prod_{k=0}^{n}\left[\sigma \widetilde{H}_{k}\right]^{\delta_{k}}=\prod_{k<m}\left[\sigma \widetilde{H}_{k}\right]^{\delta_{k}}
$$

remains as a constant on the support of $\psi_{m, i}(s)$; thus

$$
\int_{0}^{1} \psi_{m, i}(s) \psi_{n-m, j}(t) \prod_{k=0}^{n}\left[\sigma \widetilde{H}_{k}\right]^{\delta_{k}} d s=0 .
$$

Similarly, if $p>m$, then

$$
\int_{0}^{1} \psi_{m, i}(s) \psi_{n-m, j}(t) \prod_{k=0}^{n}\left[\sigma \widetilde{H}_{k}\right]^{\delta_{k}} d t=0 .
$$

For the remaining case, we have $p \leq m, q \geq m$, and $p<q$. Thus,

$$
J_{m}=\sum_{A_{m}} \int_{0}^{1} \int_{0}^{1} \psi_{m, i}(s) \psi_{n-m, j}(t) \cdot \sigma \widetilde{H}_{p} \sigma \widetilde{H}_{q} \cdot \prod_{l=p+1}^{q-1}\left[1+\sigma \widetilde{H}_{l}\right] d s d t,
$$

where $A_{m}=\{(p, q): p<q ; p \leq m, q \geq m\}$.

Note that $\widetilde{H}_{p} \widetilde{H}_{q}$ is the sum of the separately supported functions

$$
\pm h_{p, i^{\prime}}(s) h_{n-p, j^{\prime}}(t) \cdot h_{q, u}(s) h_{n-q, v}(t)
$$


each of which either equals 0 , or equals $\pm h_{q, u}(s) h_{n-p, j^{\prime}}(t)$, supported on a $2^{-q}$ by-2 $2^{-(n-p)}$ rectangle. Also note that the support of $\psi_{m, i}(s) \psi_{n-m, j}(t)$ is a $2^{-m}$-by$2^{-(n-m)}$ rectangle. So, there are no more than $2^{q-p}$ choices of $h_{p, i^{\prime}}(s) h_{n-p, j^{\prime}}(t)$. $h_{q, u}(s) h_{n-q, v}(t)$ whose support overlaps that of $\psi_{m, i}(s) \psi_{n-m, j}(t)$. Once it overlaps, the support is a $2^{-q}$-by- $2^{-(n-p)}$ rectangle, on which

$$
\prod_{l=p+1}^{q-1}\left[1+\sigma \widetilde{H}_{l}\right]
$$

is a positive constant no larger than $(1+\sigma)^{q-p-1}$.

By (2.4), we have

$$
\begin{aligned}
& \left|\int_{0}^{1} \int_{0}^{1} \psi_{m, i}(s) \psi_{n-m, j}(t) \cdot h_{q, u}(s) h_{n-p, j^{\prime}}(t) d s d t\right| \\
\leq & 2^{-2(q-m)-1} 2^{-3 m / 2} \cdot 2^{-2(m-p)-1} 2^{-3(n-m) / 2} \\
= & 2^{-2(q-p)-2} 2^{-3 n / 2} .
\end{aligned}
$$

Thus,

$$
\begin{aligned}
\left|J_{m}\right| & \leq \sigma^{2} \sum_{A_{m}} 2^{-2(q-p)-2} 2^{-3 n / 2} \cdot 2^{q-p} \cdot(1+\sigma)^{q-p-1} \\
& =\sigma^{2} \sum_{A_{m}} 2^{-3 n / 2-1} \cdot\left(\frac{1+\sigma}{2}\right)^{q-p-1} \\
& \leq \frac{(1+\sigma) \sigma^{2}}{(1-\sigma)^{2}} 2^{-3 n / 2+1} .
\end{aligned}
$$

Therefore

$$
\left|I_{3}\right| \leq \frac{(1+\sigma) \sigma^{2}}{(1-\sigma)^{2}} 2^{-3 n / 2+1} \sum_{m=0}^{n} \sum_{i=1}^{2^{m}} \sum_{j=1}^{2^{n-m}}\left|\alpha_{m, i, j}\right| .
$$

Choose $\sigma=2^{-9}$; we have $\left|I_{3}\right| \leq I_{2} / 2$. Therefore, we have

$$
\sup _{0 \leq s, t \leq 1} Y_{n}(s, t) \geq \frac{1}{2} I_{2}=2^{-3 n / 2-16} \sum_{m=0}^{n} \sum_{i=1}^{2^{m}} \sum_{j=1}^{2^{n-m}}\left|\alpha_{m, i, j}\right|,
$$

which finishes the proof of Theorem 2.1.

The final step to show Theorem 1.1 is now relatively easy. For small $\varepsilon>0$, choose the largest $n$ such that $\varepsilon 2^{3 n / 2+16} \leq(n+1) 2^{n-1}$, which implies $(n+1) 2^{n} \approx$ $c(\log (1 / \varepsilon))^{3} \varepsilon^{-2}$. Putting things together and using exponential Markov inequality, 
we have

$$
\begin{aligned}
\mathbb{P}\left(\sup _{0 \leq s, t \leq 1}|S(s, t)| \leq \varepsilon\right) \\
\quad \leq \mathbb{P}\left(\sup _{0 \leq s, t \leq 1}\left|X_{n}(s, t)\right| \leq \varepsilon\right) \\
\quad \leq \mathbb{P}\left(2^{-3 n / 2-16} \sum_{m=0}^{n} \sum_{i=1}^{2^{m}} \sum_{j=1}^{2^{n-m}}\left|g_{m, i, j}\right| \leq \varepsilon\right) \\
\quad \leq \mathbb{P}\left(\sum_{m=0}^{n} \sum_{i=1}^{2^{m}} \sum_{j=1}^{2^{n-m}}\left|g_{m, i, j}\right| \leq(n+1) 2^{n-1}\right) \\
\quad=\mathbb{P}\left(\exp \left\{-\lambda \sum_{m=0}^{n} \sum_{i=1}^{2^{m}} \sum_{j=1}^{2^{n-m}}\left|g_{m, i, j}\right|\right\} \geq \exp \left\{-\lambda(n+1) 2^{n-1}\right\}\right) \\
\leq \exp \left\{\lambda(n+1) 2^{n-1}\right\} \cdot(\mathbb{E} \exp \{-\lambda|g|\})^{(n+1) 2^{n}} \\
\leq e^{-\lambda(n+1) 2^{n} / 6} \approx e^{-C^{\prime} \varepsilon^{-2}(\log 1 / \varepsilon)^{3}}
\end{aligned}
$$

for a suitable choice of the constant $\lambda>0$, where in the last inequality we used

$$
\mathbb{E} \exp (-\lambda|g|)<e^{-2 \lambda / 3}
$$

for $\lambda>0$ sufficiently small. This can be seen by letting $h(\lambda)=\mathbb{E} \exp (2 \lambda / 3-\lambda|g|)$ and checking the derivative at $0: h^{\prime}(0)=2 / 3-(2 / \pi)^{1 / 2}<0$.

\section{Small BALl estimates UNDER The $L_{2}$-NORM}

Arguably, the $L_{2}$ norm and sup norm are the two most important norms under which the small deviation of a Gaussian field is studied. Of course, the $L_{2}$-norm case is much simpler, in general due to the Karhunen-Loéve expansion and various analytical methods which enable one to determine even the exact small deviation rate $\mathbb{P}\left(\|X\|_{2} \leq \varepsilon\right)$ as $\varepsilon \rightarrow 0$ and sometimes a closed form of the Laplace transform $\mathbb{E} \exp \left\{-\lambda\|X\|_{2}^{2}\right\}$ for all $\lambda>0$. For the sup-norm, as we have seen from Section 2 , the nice orthogonality of the eigenfunctions in the Karhunen-Loéve expansion is less useful and the small deviation rate depends not only on the eigenvalues but also heavily on the structure of eigenfunctions. Here in this section, we prove Theorem 1.2 which holds for all $d \geq 2$ with the exact constants at the logarithmic level. More precise estimates (next term in the asymptotics) can be obtained based on our approach given below, but they are less interesting and require complicated (but more or less routine) calculations. So we omit them.

Let us consider the one-dimensional Slepian process first, namely

$$
S(t)=W(t+a)-W(t), \quad 0 \leq t \leq 1,
$$

with $a \geq 1$ fixed, where $W(t)$ is the standard Brownian motion. Let us first consider the Karhunen-Loéve expansion in a one-dimensional setting.

Proposition 3.1. If $a \geq 1$, then we have in distribution (as process)

$$
S(t)=W(t+a)-W(t)=\sum_{n \geq 1} \sqrt{\lambda_{n}^{(a)}} \xi_{n} \phi_{n}(t), \quad \lambda_{n}^{(a)}>0,
$$


where $\xi_{n}$ are independent standard normal random variables,

$$
\left(2 \lambda_{2 n}^{(a)}\right)^{-1 / 2}=(n-1) \pi+\pi / 2
$$

and $\left(2 \lambda_{2 n-1}^{(a)}\right)^{-1 / 2}$ are the only solutions of the equation

$$
(2 a-1) \tan x=x^{-1}, \quad a \geq 1, \quad \text { on } \quad[(n-1) \pi,(n-1) \pi+\pi / 2) .
$$

In particular, we have in distribution

$$
\int_{0}^{1} S^{2}(t) d t=\int_{0}^{1}(W(t+a)-W(t))^{2} d t=\sum_{n \geq 1} \lambda_{n}^{(a)} \xi_{n}^{2} .
$$

Moreover, we have the Laplace transform

$$
L(\lambda)=\mathbb{E} e^{-\lambda \int_{0}^{1} S^{2}(t) d t}=[\cosh \sqrt{\lambda}(\cosh \sqrt{\lambda}+(2 a-1) \sqrt{\lambda} \sinh \sqrt{\lambda})]^{-1 / 2} .
$$

Proof. By direct calculation, we have the covariance function

$$
K(s, t)=\mathbb{E} S(s) S(t)=a-|s-t| \quad \text { for } \quad s, t \in[0,1] .
$$

To find the eigenvalues associated with this covariance function, we need to solve the integral equation

$$
\lambda f(t)=\int_{0}^{1} K(s, t) f(s) d s, \quad 0 \leq t \leq 1 .
$$

That is, for $a \geq 1$,

$$
\lambda f(t)=\int_{0}^{t}(a-t+s) f(s) d s+\int_{t}^{1}(a+t-s) f(s) d s, \quad 0 \leq t \leq 1 .
$$

We may differentiate (3.3) with respect to $t$ to obtain

$$
\lambda f^{\prime}(t)=-\int_{0}^{t} f(s) d s+\int_{t}^{1} f(s) d s .
$$

Differentiate again to obtain $\lambda f^{\prime \prime}(t)=-2 f(t)$. Hence

$$
f(t)=c_{1} \sin \sqrt{2 \lambda^{-1}} t+c_{2} \cos \sqrt{2 \lambda^{-1}} t .
$$

Setting $t=0$ and $t=1$ in (3.3) and (3.4), we obtain boundary conditions

$$
f^{\prime}(0)+f^{\prime}(1)=0 \quad \text { and } \quad f(0)+f(1)=(2 a-1) f^{\prime}(0) .
$$

Substituting (3.5) into (3.6) and simplifying yields

$$
\begin{aligned}
\left(1+\cos \sqrt{2 \lambda^{-1}}\right) c_{1}-\sin \sqrt{2 \lambda^{-1}} c_{2} & =0 \\
\left(\sin \sqrt{2 \lambda^{-1}}-(2 a-1) \sqrt{2 \lambda^{-1}}\right) c_{1}+\left(1+\cos \sqrt{2 \lambda^{-1}}\right) c_{2} & =0 .
\end{aligned}
$$

In order for there to be non-zero choices for $c_{1}$ and $c_{2}$, the determinant of the above two equations has to be zero. Thus,

$$
\left(2+2 \cos \sqrt{2 \lambda^{-1}}-(2 a-1) \sqrt{(2 \lambda)^{-1}} \sin \sqrt{2 \lambda^{-1}}\right)=0 .
$$

Simplifying, we obtain

$$
\cos \sqrt{(2 \lambda)^{-1}}\left(\cos \sqrt{(2 \lambda)^{-1}}-(2 a-1) \sqrt{(2 \lambda)^{-1}} \sin \sqrt{(2 \lambda)^{-1}}\right)=0 .
$$


By a general theorem in Gao et al. G-03, we have the Laplace transform of $\|S\|_{2}^{2}$ in the closed form given in (3.2).

To prove Theorem 1.2, we note that by using the inequality $\tan x>x$ on $(0, \pi / 2)$ and (3.1), we have

$$
\begin{aligned}
& (2 a-1)^{-1}\left(2 \lambda_{2 n-1}^{(a)}\right)^{1 / 2}=\tan \left(\left(2 \lambda_{2 n-1}^{(a)}\right)^{-1 / 2}\right) \\
= & \tan \left(\left(2 \lambda_{2 n-1}^{(a)}\right)^{-1 / 2}-(n-1) \pi\right) \\
> & \left(2 \lambda_{2 n-1}^{(a)}\right)^{-1 / 2}-(n-1) \pi \geq 0,
\end{aligned}
$$

which gives us $\left(2 \lambda_{2 n-1}\right)^{-1 / 2}=(n-1) \pi+O(1 / n)$. Together with the expression for $\lambda_{2 n}$, we have

$$
\lambda_{n} \sim 2 \pi^{-2} n^{-2} .
$$

The proof of Theorem 1.2 then follows from the method used in Example 3 in $\mathrm{Li}$ Li92. Of course, arguments in Karol, Nazarov and Nikitin K-03, Fill and Torcaso FT04, and Gao and Li GL04 can also be used to finish the proof. We omit the details.

\section{ACKNOWLEDGEMENTS}

The authors would like to thank the referee for a careful reading of the paper and for useful comments.

\section{REFERENCES}

[Ad84] Adler, R.J. (1984). The supremum of a particular Gaussian field, Ann. Prob. 12, 436-444. MR0735847 (86a:60058)

[Ba88] Bass, R.F. (1988). Probability estimates for multiparameter Brownian processes. Ann. Prob. 16, 251-264. MR0920269 (89b:60103)

[Be98] Belinsky, E. (1998). Estimates of entropy numbers and Gaussian measures for classes of functions with bounded mixed derivative. J. Approx. Th. 93, 114-127. MR.1612794 (2000c:41032)

[BL02] Belinsky, E. and Linde, W. (2002). Small ball probabilities of fractional Brownian sheets via fractional integration operators. J. Theoret. Probab. 15, 589-612. MR 1922439 (2004d:60092)

[Cs82] Csáki, E. (1982). On small values of the square integral of a multiparameter Wiener process. Statistics and Probability. Proc. of the 3rd Pannonian Symp. on Math. Stat. D. Reidel Publishing Company, pp. 19-26. MR0758997

[Du00] Dunker, T. (2000). Estimates for the small ball probabilities of the fractional Brownian sheet. J. Theor. Probab. 13, 357-382. Erratum: J. Theor. Probab. 14, (2001), 607. MR.1777539 (2001g:60085) MR:1838746 (2002e:60054)

[D-99] Dunker, T., Kuhn, T., Lifshits, M. A. and Linde, W. (1999). Metric entropy of integration operators and small ball probabilities for the Brownian sheet. J. Approx. Theory 101, 63-77. MR.1724026(2001d:60032)

[FT04] Fill, J. and Torcaso, F. (2004). Asymptotic Analysis via Mellin Transforms for Small Deviations in $L^{2}$-norm of Integrated Brownian Sheets. Probab. Theo. and Related Fields 130, 259-288. MR2093764 (2005i:60066)

[G-03] Gao, F., Hannig, J., Lee, T.-Y. and Torcaso, F. (2003). Laplace transforms via Hadamard factorization, Electron. J. Probab., 8, no. 13, 1-20. MR.1998764(2005h:60110)

[GL04] Gao, F. and Li, W.V. (2004). Logarithmic level comparison for small deviation probabilities, J. Theory Probab., 2006, DOI 10.1007/s10959-006-0026-1 (online).

[K-03] A. Karol, A. Nazarov and Ya. Nikitin (2003), Tensor products of compact operators and logarithmic $L_{2}$-small ball asymptotics for Gaussian random fields, Preprint. 
[KL93] Kuelbs, J. and Li, W.V. (1993). Metric entropy and the small ball problem for Gaussian measures. J. Funct. Anal. 116, 133-157. MR1237989 (94j:60078)

[Li92] Li, W.V. (1992). Comparison results for the lower tail of Gaussian seminorms, J. Theoret. Probab, 5, 1-31. MR.1144725 (93k:60088)

[LL99] Li, W.V. and Linde, W. (1999). Approximation, metric entropy and small ball estimates for Gaussian measures. Ann. Probab. 27, 1556-1578. MR1733160 (2001c:60059)

[LS01] Li, W.V. and Shao, Q.-M. (2001). Gaussian processes: inequalities, small ball probabilities and applications. Stochastic processes: theory and methods, Handbook of Statist., Vol. 19, 533-597. MR1861734

[LT86] Lifshits, M.A., and Tsyrelson, B.S. (1986). Small ball deviations of Gaussian fields. Theor. Probab. Appl. 31, 557-558.

[Ma04] Martin, A. (2004). Small ball asymptotics for the stochastic wave equation. J. Theoret. Probab. 17, 693-703. MR2091556 (2005h:60188)

[Ta94] Talagrand, M. (1994). The small ball problem for the Brownian sheet. Ann. Probab. 22, 1331-1354. MR:1303647 (95k:60049)

[Te95] Temlyakov, V. (1995). An inequality for trigonometric polynomials and its application for estimating the entropy numbers. Journal of Complexity 11, 293-307. MR.1334238 (96c:41052)

Department of Mathematics, University of Idaho, Moscow, Idaho 83844

E-mail address: fuchang@uidaho.edu

Department of Mathematical Sciences, University of Delaware, Newark, Delaware 19716

E-mail address: wli@math.udel.edu 